\title{
Activity of DNA glycosylases on non-canonical DNA substrates
}

\author{
Evgeniia Diatlova \\ Novosibirsk State University, Novosibirsk, Russia \\ ICBFM SB RAS, Novosibirsk, Russia \\ jannie.lapt@gmail.com
}

\author{
Dmitry Zharkov \\ Novosibirsk State University, Novosibirsk, Russia \\ ICBFM SB RAS, Novosibirsk, Russia \\ dzharkov@niboch.nsc.ru
}

\begin{abstract}
Until recently, DNA repair was almost exclusively considered in the B-DNA context, since noncanonical DNA was not believed to be a significant target for damage in the bulk genome. A paradigm shift that followed the discoveries of functionally important roles of non-canonical DNA and the cases of apparent connection of regulation with DNA damage put DNA repair in non-canonical structures into the focus of active studies. In this work we investigated base excision repair in non-canonical DNA structures involving hairpins, quadruplexes and DNA/RNA heteroduplexes.
\end{abstract}

Keywords - DNA glycosylase, non-canonical DNA, base excision repair.

\section{Introduction}

B-form is the main conformation adopted by doublestranded DNA under physiological salt, $\mathrm{pH}$, and temperature conditions. Although genomic DNA in living cells mostly exists in the B-form, it became clear over the past decades that DNA exhibits significant conformational polymorphism and that many functionally important genome elements can assume alternative structures in vivo, either permanently or temporarily. Such non-B-DNA structures (thereafter referred to as "noncanonical DNA") include cruciform DNA, hairpins, triplexes, quadruplexes, intercalated motifs (i-motifs), singlestranded DNA, Z-DNA, RNA/DNA heteroduplexes, and displacement loops (D-loops) containing either RNA (Rloops) or DNA invading strands. These elements are often involved in gene activity regulation and genome stabilization, or, on the contrary, could be intermediates of deleterious processes causing genome instability.

As a chemically active molecule, DNA is in a constant flux of spontaneous and directed modification. Notably, most noncanonical DNA forms is more sensitive to DNA damage than B-DNA, Recently, some non-canonical DNA, such as quadruplexes, were shown to serve as oxidation-dependent transcriptional regulatory elements in human cells [1-3]. On the other hand, non-canonical structures, including DNA hairpins and Z-DNA, turned out to be associated with severe human diseases due to their mutation-prone nature and gene activity misregulation [4-5].

\section{Methods}

In this work we investigated the ability of mammalian OGG1, NEIL1 and NEIL2 DNA glycosylases; human and E. coli uracil-DNA glycosylase; E. coli and Lactococcus lactis formamidopyrimodine-DNA glycosylase; and E. coli MutY, Nei and Nth DNA glycosylases to process substratres other than double-stranded DNA. Oligonucleotide substrates containing one of damaged bases (oxoguanine, 5hydroxyuracil, 5,6-dihydrouracil, uracil, AP site) were annealed with complementary DNA (forming one base bulge substrate), or RNA strand (forming DNA/RNA heteroduplexes with each of four nucleotides in the opposite position). For uracil-glycosylases we also prepared oligonucleotides containing uracil and G-quadruplex.

RESULTS

We performed a screen of activity for all enzymesubstrate pairs. Both human and E. coli uracil-DNAglycosylases removed uracil from structures containing Gquadruplex, one base bulges and DNA/RNA heteroduplexes regardless of the opposite nucleotide. There was no activity of MutY. Enzymes of the helix-two-turn-helix (H2TH) structural superfamily (Fpg from E. coli and Lactococcus lactis, Nei E. coli and mouse NEIL1 and NEIL2) all cleaved DNA containing oxoguanine, 5-hydroxyuracil, 5,6dihydrouracil, AP site in any context, but Nei and NEILs removed oxoguanine relatively slight. Nei also showed small activity on the substrate with a bulged uracil. Nth removed all damage except uracil and hydroxyuracil. OGG1 processed substrates with oxoguanine from bulge and when $\mathrm{C}$ or $\mathrm{U}$ were opposite in RNA.

We also obtained kinetic data of Fpg, OGG1, human and E.coli UNG on substrates with one base bulges, and also on DNA containing G-quadruplex for uracil glycosylases.

\section{ACKNOWLEDGMENT}

This work was supported by Russian Science Foundation (No. 17-14-01190)

\section{REFERENCES}

[1] Zarakowska E., Gackowski D., Foksinski M., Olinski R. (2014) Are 8oxoguanine (8-oxoGua) and 5-hydroxymethyluracil (5-hmUra) oxidatively damaged DNA bases or transcription (epigenetic) marks? Mutat. Res., 764-765, p. 58-63.

[2] Fleming A.M., Burrows C.J. (2017) 8-Oxo-7,8-dihydroguanine, friend and foe: Epigenetic-like regulator versus initiator of mutagenesis. DNA Repair, 56, p. 75-83.

[3] Seifermann M., Epe B. (2017) Oxidatively generated base modifications in DNA: Not only carcinogenic risk factor but also regulatory mark? Free Radic. Biol. Med., 107, p. 258-265.

[4] (McMurray C.T. (2010) Mechanisms of trinucleotide repeat instability during human development. Nat. Rev. Genet., 11, p. 786-799.

[5] Zhao X., Krishnamurthy N., Burrows C.J., David S.S. (2010a) Mutation versus repair: NEIL1 removal of hydantoin lesions in singlestranded, bulge, bubble, and duplex DNA contexts. Biochemistry, 49, p. $1658-1666$ 